\title{
Erratum
}

\section{Erratum to "Note on Some Nonlinear Integral Inequalities and Applications to Differential Equations"}

\author{
Khaled Boukerrioua \\ Department of Mathematics, University of 08 Mai 1945, BP 401, 24000 Guelma, Algeria \\ Correspondence should be addressed to Khaled Boukerrioua, khaledv2004@yahoo.fr \\ Received 7 May 2012; Accepted 19 June 2012 \\ Copyright (C) 2012 Khaled Boukerrioua. This is an open access article distributed under the \\ Creative Commons Attribution License, which permits unrestricted use, distribution, and \\ reproduction in any medium, provided the original work is properly cited.
}

First, we apologize for the misprints in the original article. Now we correct those misprints.

Page 1, line 2: Between equations and The Gronwall-Bellman insert "Among various types of integral inequalities."

Page 1, line 5: Replace diferential by differential.

Page 2, line 1: Replace of the situation by to the situation.

Page 2, Lemma 2.1: between For $x \in \mathbb{R}_{+}, y \in \mathbb{R}_{+}, 1 / p+1 / q=1$, and one has insert "with $p>1 . "$

Page 2, Theorem 2.3: Replace and there by "If there."

Page 2, Theorem 2.3: Replace and $u(t)$ satisfy by and the function $u(t)$ satisfies.

Page 3, Proof of Theorem 2.3, line 9: Replace it yields by we obtain.

Page 4, Remark 2.4, line 2: Replace become by becomes.

Page 4, Theorem 2.5, line 1: Replace holds by hold.

Page 4, Theorem 2.5, (2.15): Replace $\int_{0}^{t} b(s) \sum_{i=1}^{i=n} h_{i}(t)\left(a(t)+p^{\star} / p\right)^{\left(p^{\star} / p\right)-1} d s$ by $\int_{0}^{t} b(s) \sum_{i=1}^{i=n} h_{i}(s)\left(a(s)+p^{\star} / p\right)^{\left(p^{\star} / p\right)-1} d s$.

Page 4, Theorem 2.5, (2.16): Replace $\int_{0}^{t} b(s) \sum_{i=1}^{i=n} h_{i}(t)\left(a(t)+p^{\star} / p\right)^{\left(p^{\star} / p\right)-1} d s$ by $\int_{0}^{t} b(s) \sum_{i=1}^{i=n} h_{i}(s)\left(a(s)+p^{\star} / p\right)^{\left(p^{\star} / p\right)-1} d s$.

Page 5,(2.28): Replace $\int_{0}^{t}\left(p^{\star} / p-1\right) b(t) \sum_{i=1}^{i=n} h_{i}(t)\left(a(t)+p^{\star} / p\right)^{\left(p^{\star} / p\right)-1} d s$ by $\int_{0}^{t}\left(p^{\star} / p-\right.$ 1) $b(s) \sum_{i=1}^{i=n} h_{i}(s)\left(a(s)+p^{\star} / p\right)^{\left(p^{\star} / p\right)-1} d s$.

Page 6, (2.29): Replace $\int_{0}^{t}\left(p^{\star} / p-1\right) b(t) \sum_{i=1}^{i=n} h_{i}(t)\left(a(t)+p^{\star} / p\right)^{\left(p^{\star} / p\right)-1} d s$ by $\int_{0}^{t}\left(p^{\star} / p-\right.$ 1) $b(s) \sum_{i=1}^{i=n} h_{i}(s)\left(a(s)+p^{\star} / p\right)^{\left(p^{\star} / p\right)-1} d s$. 
Page 6, (2.30): Replace $\int_{0}^{t}\left(p^{\star} / p-1\right) b(t) \sum_{i=1}^{i=n} h_{i}(t)\left(a(t)+p^{\star} / p\right)^{\left(p^{\star} / p\right)-1} d s$ by $\int_{0}^{t}\left(p^{\star} / p-\right.$ 1) $b(s) \sum_{i=1}^{i=n} h_{i}(s)\left(a(s)+p^{\star} / p\right)^{\left(p^{\star} / p\right)-1} d s$.

Page 6, (2.31): Replace $\int_{0}^{t} b(t) \sum_{i=1}^{i=n} h_{i}(t)\left(a(t)+p^{\star} / p\right)^{\left(p^{\star} / p\right)-1} d s \quad$ by $\int_{0}^{t} b(s) \sum_{i=1}^{i=n} h_{i}(s)\left(a(s)+p^{\star} / p\right)^{\left(p^{\star} / p\right)-1} d s$.

Page 6, (2.33): Replace $\int_{0}^{t} b(t) \sum_{i=1}^{i=n} h_{i}(t)\left(a(t)+p_{\star} / p\right)^{\left(p^{\star} / p\right)-1} d s \quad$ by $\int_{0}^{t} b(s) \sum_{i=1}^{i=n} h_{i}(s)\left(a(s)+p_{\star} / p\right)^{\left(p^{\star} / p\right)-1} d s$.

Page 6, (2.34): Replace $\int_{0}^{t} b(s) \sum_{i=1}^{i=n} h_{i}(t)\left(a(t)+p_{\star} / p\right)^{\left(p^{\star} / p\right)-1} d s \quad$ by $\int_{0}^{t} b(s) \sum_{i=1}^{i=n} h_{i}(s)\left(a(s)+p_{\star} / p\right)^{\left(p^{\star} / p\right)-1} d s$.

Page 6, in Theorem 2.6: Replace holds by hold.

Page 6, in Proof: Replace for by For.

Page 7, in Remark 2.7: Replace if by If.

Page 7, in Theorem 2.8: Replace holds by hold.

Page 7, in Theorem 2.8 line 2: Replace nonnegative by positive.

Page 8, in Theorem 2.9: Replace $\partial / \partial s k(t, s)$ by $(\partial / \partial s) k(t, s)$.

Page 12, line 3: Replace tacking account by Taking into account.

Page 12, in Remark 2.10: Replace if by If.

Page 11, (2.63): Replace $\int_{0}^{t}(\partial / \partial s) k(t, s)\left(a(s)+p^{\star} / p+b(s) v(s)\right)^{p^{\star} / p} \quad$ by $\int_{0}^{t}(\partial / \partial s) k(t, s) \sum_{i=1}^{i=n} h_{i}(s)\left(a(s)+p^{\star} / p+b(s) v(s)\right)^{p^{\star} / p}$.

Page 12, (3.1): Insert $t \geq t_{0}$.

Page 12 in (3.2): Replace $\left\{c+\int_{t_{0}}^{t} f(\tau)\left[((q / p) c+(p-q) / p) \exp \int_{t_{0}}^{t} A^{*}(s) d s+\right.\right.$ $\left.\left.\int_{t_{0}}^{t} B^{*}(s)\left(\exp \int_{s}^{t} A^{*}(\sigma) d \sigma\right) d s\right] d \tau\right\}^{1 / p}$ by $\left\{c+\int_{t_{0}}^{t} f(\tau)[((q / p) c+(p-\right.$ $\left.\left.q) / p) \exp \int_{t_{0}}^{\tau} A^{*}(s) d s+\int_{t_{0}}^{\tau} B^{*}(s)\left(\exp \int_{s}^{\tau} A^{*}(\sigma) d \sigma\right) d s\right] d \tau\right\}^{1 / p}, t \geq t_{0}$.

Page 13, Proof of Theorem 3.1, in (3.4), (3.5), (3.6), and (3.7): Replace $Z(t)$ by $z(t)$.

Page 13 between (3.7) and (3.8): Replace its follow by it follows.

Page 14, in application, line 2: Replace equation by equations.

Page 14, in end of the Section 3: Replace then the result required is found by: using (3.13) in (3.14), we get the required inequality in (3.2).

Page 14 in (3.13): Replace $z(t) \leq c+\int_{t_{0}}^{t} f(\tau)\left[((q / p) c+(p-q) / p) \exp \int_{t_{0}}^{t} A^{*}(s) d s+\right.$ $\left.\int_{t_{0}}^{t} B^{*}(s)\left(\exp \int_{s}^{t} A^{*}(\sigma) d \sigma\right) d s\right] d \tau$, by $z(t) \leq c+\int_{t_{0}}^{t} f(\tau)[((q / p) c+(p-$ q) $\left./ p) \exp \int_{t_{0}}^{\tau} A^{*}(s) d s+\int_{t_{0}}^{\tau} B^{*}(s)\left(\exp \int_{s}^{\tau} A^{*}(\sigma) d \sigma\right) d s\right] d \tau$.

Page 14, in (4.1) insert $t>0$.

Page 14, in (4.2): Replace $\sum_{i=1}^{i=n} h_{i}(t) u^{p_{i}}(t)$ by $\sum_{i=1}^{i=n} h_{i}(t)|u|^{p_{i}}$.

Page 14, in Example 4.1: Replace $p, p_{i}(i=1, \ldots, n) \geq 0$ by $p, p_{i}(i=1, \ldots, n)>0$.

Page 15, line 2: we estimate the solution $u(t)$ of (4.1). 
International Journal of Differential Equations

Page 15 in Proof of Theorem 4.3: Replace (4.5) by this formula $u(t) \leq\left\{|c|^{p}+\right.$ $\left.\int_{t_{0}}^{t} f(\tau)\left[\left((q / p)|c|^{p}+(p-q) / p\right) \exp \int_{t_{0}}^{\tau} A^{*}(s) d s+\int_{t_{0}}^{\tau} B^{*}(s)\left(\exp \int_{s}^{\tau} A^{*}(\sigma) d \sigma\right) d s\right] d \tau\right\}^{1 / p}$.

Page 15 in Proof of Theorem 4.3: Replace $u^{p}(t)=c^{p}+\int_{t_{0}}^{t} f(\tau)\left[u^{q}(\tau)+\right.$ $\left.\int_{t_{0}}^{t} k(\tau, s) u^{r}(s) d s\right] d \tau$ by $u^{p}(t)=c^{p}+\int_{t_{0}}^{t} f(\tau)\left[u^{q}(\tau)+\int_{t_{0}}^{\tau} k(\tau, s) u^{r}(s) d s\right] d \tau$.

Page 15, Proof of Theorem 4.3: Replace (4.7) by $|u(t)|^{p} \leq|c|^{p}+\int_{t_{0}}^{t} f(\tau)\left[|u(\tau)|^{q}+\right.$ $\left.\int_{t_{0}}^{\tau} k(\tau, s)|u(\tau)|^{r} d s\right] d \tau, t_{0} \leq s \leq \tau \leq t$.

End of the corrigendum. 


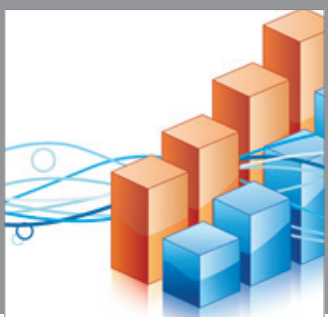

Advances in

Operations Research

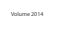

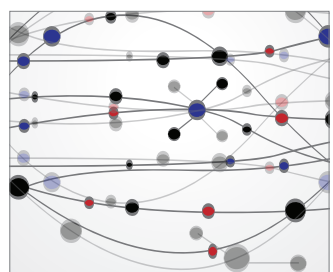

\section{The Scientific} World Journal
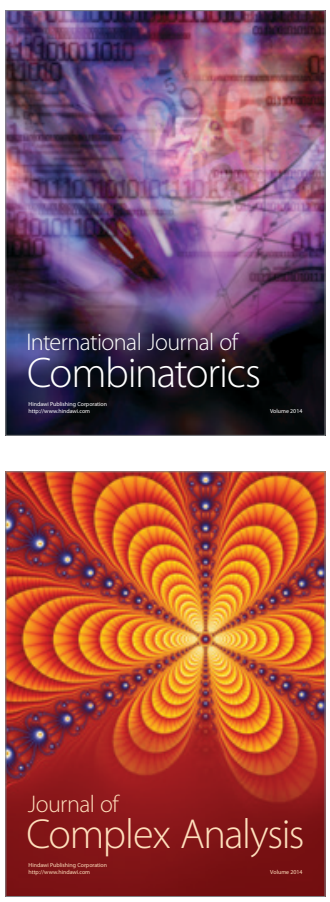

International Journal of

Mathematics and

Mathematical

Sciences
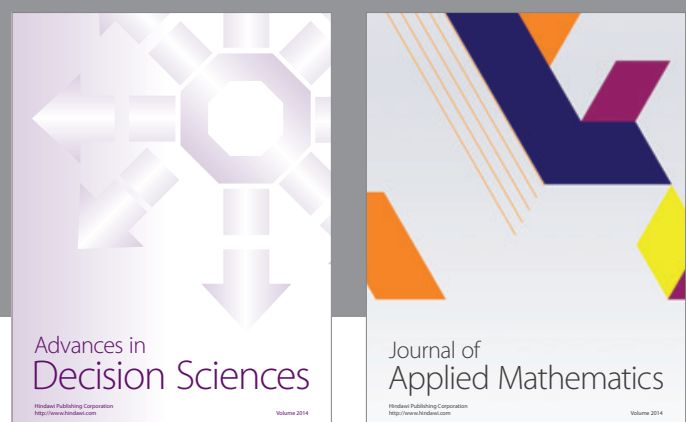

Journal of

Applied Mathematics
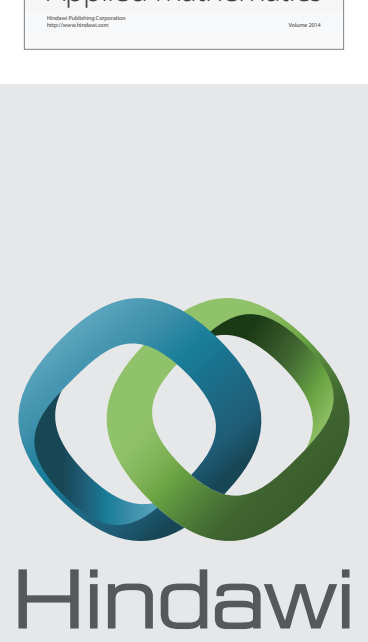

Submit your manuscripts at http://www.hindawi.com
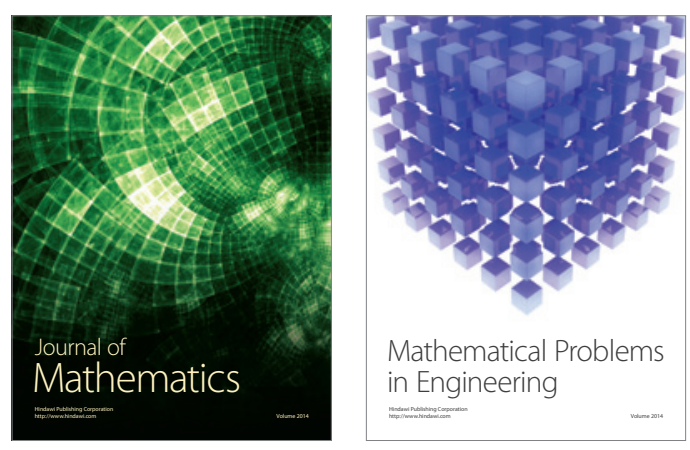

Mathematical Problems in Engineering
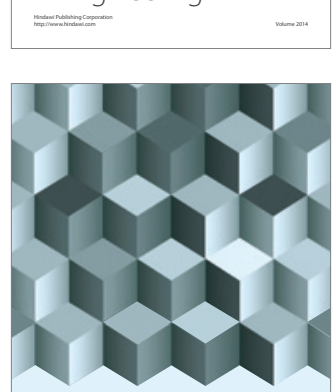

Journal of

Function Spaces
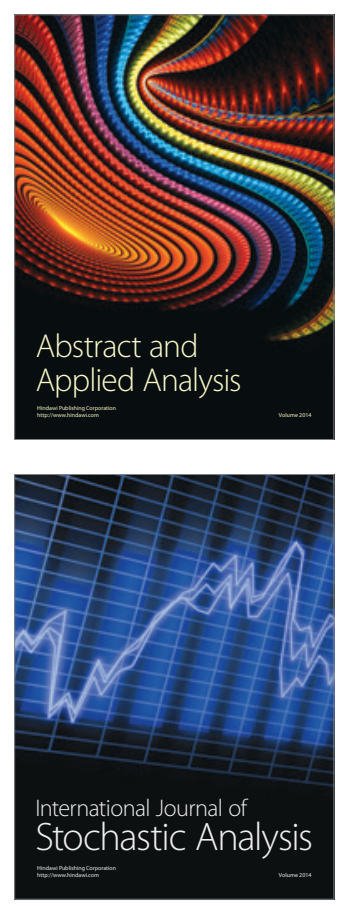

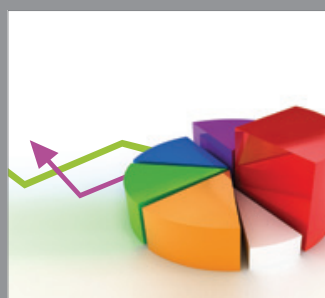

ournal of

Probability and Statistics

Promensencen
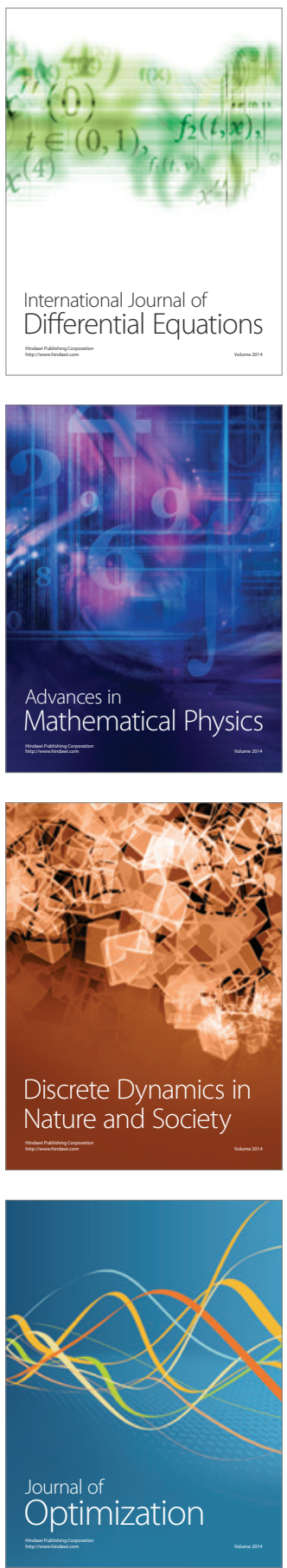